\title{
Dual Matrix and Biquaternion Methods of Solving Direct and Inverse Kinematics Problems of Manipulators for Example Stanford Robot Arm. II
}

\section{E. I. Lomovtseva, Yu. N. Chelnokov}

Saratov State University, 83, Astrahanskaya str., 410012, Saratov, Russia, LomovtsevaEl@yandex.ru, chelnokovyun@info.sgu.ru The methology of solving the inverce kinematics problem of manipulators by using biquaternion theory of kinematics control is shown on the example of Stanford robot arm. Solving of the inverce kinematics problem of Stanford robot arm is performed using the simplest control law. The analysis of numerical solution results is made. The efficacy of applying the theory of kinematics control for solving the inverce kinematics problem of manipulators is proved. Dual matrix and biquaternion methods of solving direct kinematics problem of manipulators were considered in [1].

Key words: robot-manipulator, direct kinematics problem, dual direction cosine matrix, biquaternion, quaternion, kinematic equations.

\section{References}

1. Lomovtseva E. I., Chelnokov Yu. N. Dual Matrix and Biquaternion Methods of Solving Direct and Inverse Kinematics Problems of Manipulators, for Example Stanford Robot Arm. I. Izv. Saratov. Univ. (N. S.), Ser. Math. Mech. Inform., 2013, vol. 13, no. 4. pp. 82-89 (in Russian).

2. Chelnokov Yu. N. Biquaternion Solution of the Kinematic Control Problem for the Motion of a Rigid Body and Its Application to the Solution of Inverse Problems of Robot-Manipulator Kinematics. Mechanics of

Solids [Izv. RAN. Mehanika tverdogo tela], 2013, vol. 48, no. 1. pp. 31-46.

3. Fu K. S., Gonzalez R. C. ,Lee C. S. G. Robotics : Control, Sensing, Vision, and Intelligence. McGraw-Hill, Inc, 1987, $580 \mathrm{p}$.

4. Chelnokov Yu. N. Kvaternionnye i bikvaternionnye modeli i metody mehaniki tverdogo tela $i$ ih prilozhenija. Geometrija dvizhenija [Quaternion and Biquaternion Models and Methods of Mechanics of a Rigid Body and their Applications. Geometry of Motion.] Saratov, Saratov Univ. Press, 2006, 236 p. (in Russian).

УДК 539.3

\section{УСТОЙЧИВОСТЬ КОНСТРУКТИВНО-ОРТОТРОПНОЙ НЕОДНОРОДНОЙ ЦИЛИНДРИЧЕСКОЙ ОБОЛОЧКИ ОТ НЕРАВНОМЕРНОЙ РАДИАЛЬНОЙ НАГРУЗКИ}

\begin{abstract}
А. А. Мочалин
Кандидат фризико-математических наук, доцент касредры прикладной математики и системного анализа, Саратовский государственный технический университет имени Гагарина Ю. А., a.mochalin@inbox.ru

На базе полубезмоментной теории В. З. Власова рассматривается задача об устойчивости цилиндрической конструктивноортотропной оболочки переменной вдоль образующей толщины при действии осесимметричного изменяющегося вдоль оси оболочки радиального давления. При одном соотношении изменения толщины и давления получено точное решение для нахождения одной из величин в законе изменения давления, при которой происходит потеря устойчивости оболочки.

Ключевые слова: цилиндрическая оболочка, теория оболочек, устойчивость оболочек, радиальная нагрузка, критическое давление, толщина оболочка, теория упругости.
\end{abstract}

1. В линейной теории цилиндрических оболочек широкое применение нашла полубезмоментная теория В. З. Власова, учитывающая особенности напряженного состояния оболочек, длина которых находится в пределах $D<l<8 D$, где $D-$ диаметр оболочки, $l-$ её длина. В основе этой теории лежат две гипотезы - статическая и геометрическая, позволяющие существенно упростить уравнения, описывающие состояние устойчивости оболочки. Считается, что удлинение в окружном направлении и углы сдвига в срединной поверхности равны нулю, полагаются равными нулю перерезывающая сила и изгибающий момент в осевом направлении, а также крутящий момент.

На основе гипотез полубезмоментной теории цилиндрических оболочек В. 3. Власова [1] рассмотрим конструктивно-ортотропную неоднородную оболочку под действием неравномерной радиальной 
нагрузки. Уравнение совместности деформаций и уравнение равновесия после несложных преобразований перепишется в виде

$$
\begin{gathered}
R \frac{\partial^{4} \varepsilon_{1}}{\partial y^{4}}+\frac{1}{R} \frac{\partial^{2} \varepsilon_{1}}{\partial y^{2}}=\frac{\partial^{2} \varkappa_{2}}{\partial x^{2}}, \\
R \frac{\partial^{4} M_{2}}{\partial y^{4}}+\frac{1}{R} \frac{\partial^{2} M_{2}}{\partial y^{2}}+\frac{\partial^{2} T_{1}}{\partial x^{2}}=P \\
P=-\frac{\partial q_{1}}{\partial x}+\frac{\partial q_{2}}{\partial y}-R \frac{\partial^{2} q_{n}}{\partial y^{2}},
\end{gathered}
$$

где $x$ - координата, откладываемая по образующей, $y-$ координата, откладываемая по дуге поперечного круга цилиндра.

Соотношения упругости с учетом допущений полубезмоментной теории В. 3. Власова запишутся в форме

$$
T_{1} \approx E h m_{1} \varepsilon_{1}, \quad M_{2}=D n^{2} n_{2} \varkappa_{2}
$$

Упругие усилия и моменты связаны с деформациями срединной поверхности соотношениями (2).

Введем безразмерные переменные $\xi=x / l, \theta=y / R(l-$ длина оболочки) и функцию $\boldsymbol{\Phi}$, связанную с компонентами поля перемещений $\bar{U}(u, v, w)$ равенствами

$$
u=-\frac{1}{l} \frac{\partial^{3} \Phi}{\partial \xi \partial \theta^{2}}, \quad v=\frac{1}{R} \frac{\partial^{2} \Phi}{\partial \theta^{2}}, \quad w=\frac{1}{R} \frac{\partial^{4} \Phi}{\partial \theta^{4}}
$$

Тогда

$$
\begin{array}{rlrl}
\varepsilon_{1} & =-\frac{1}{l^{2}} \frac{\partial^{4} \Phi}{\partial \xi^{2} \partial \theta^{2}}, & \varkappa_{2}=-\frac{1}{R^{3}}\left(\frac{\partial^{4} \Phi}{\partial \theta^{4}}+\frac{\partial^{6} \Phi}{\partial \theta^{6}}\right), \\
T_{1}=-\frac{E h}{l^{2}} \frac{\partial^{4} \Phi}{\partial \xi^{2} \partial \theta^{2}}, & M_{2}=-\frac{D}{R^{3}}\left(\frac{\partial^{6} \Phi}{\partial \theta^{6}}+\frac{\partial^{4} \Phi}{\partial \theta^{4}}\right) .
\end{array}
$$

Подстановка (3) тождественно удовлетворяет уравнению (1), а уравнение (2) перепишется в следующем виде:

$$
\frac{\partial^{2}}{\partial \xi^{2}}\left[h(\xi) \frac{\partial^{2} \Phi}{\partial \xi^{2}}\right]+\frac{D l^{4}}{E R^{6}} \Omega \Omega \Phi+\frac{l^{4}}{E} \int_{0}^{\theta} \int_{0}^{\theta} P^{*} d \theta d \theta=0
$$

Здесь $P^{*}=-\frac{1}{l} \frac{\partial q_{1}}{\partial \xi}+\frac{1}{R}\left(\frac{\partial q_{2}}{\partial \theta}-\frac{\partial^{2} q_{n}}{\partial \theta^{2}}\right)$, а $\Omega=\frac{\partial^{2}}{\partial \theta^{2}}\left(1+\frac{\partial^{2}}{\partial \theta^{2}}\right)-$ дифференциальный оператор В. З. Власова.

Уравнением (4) будем пользоваться при исследовании устойчивости цилиндрических оболочек средней и большой длины под действием радиальной нагрузки и интегрировать при граничных условиях, зависящих от способа закрепления торцов оболочки.

2. Пусть оболочка находится под действием неравномерной радиальной нагрузки $q=q_{0}(1+\alpha \xi)^{-6}$. Компонента нагрузки $q_{n}$ в этом случае определится выражением $q_{n}=-q_{0} R \varkappa_{2}(1+\alpha \xi)^{-6}$, где параметр $q_{0}$ подлежит определению.

Выбирая функцию $\Phi$ в форме $\Phi=X(\xi) \sin k \theta$, где $k-$ число волн вдоль окружности оболочки при потере устойчивости, и задавая закон изменения толщины в виде $h(\xi)=h_{0}(1+\alpha \xi)^{-2}$, приходим к уравнению

$$
\frac{d^{2}}{d \xi^{2}}\left[(1+\alpha \xi)^{-2} \frac{d^{2} X}{d \xi^{2}}\right]-\lambda^{4}(1+\alpha \xi)^{-6} X=0
$$

где

$$
\lambda^{4}=\frac{q_{0} k^{4} l^{4}}{E R^{3} h_{0}}\left(k^{2}-1\right)-\frac{l^{4} h_{0}^{2}\left(k^{2}-1\right) k^{4}}{12 R^{6}\left(1-v^{2}\right)} .
$$

Уравнение (5) после введения новой переменной $z=\ln (1+\alpha \xi)$ и новой функции $W(z)$ соотношением $X=W e^{-z / 6}$ может быть приведено к дифференциальному уравнению четвертого порядка с 
постоянными коэффициентами:

$$
W^{(4)}-\frac{10}{3} W^{(3)}+\frac{25}{6} W^{(2)}+\frac{89}{54} W^{\prime}-\left(\frac{\lambda^{4}}{\alpha^{4}}-\frac{145}{1296}\right) W=0,
$$

характеристическое уравнение для которого запишется в виде

$$
\left(\delta^{2}+a \delta+c\right)\left(\delta^{2}+b \delta+d\right)=0
$$

где величины $a, b, c, d$ связаны равенствами

$$
a+b=-\frac{10}{3}, \quad a b+c+d=\frac{25}{6}, \quad b c+d a=\frac{89}{54}, \quad c d=\frac{145}{1296}-\frac{\lambda^{4}}{\alpha^{4}}
$$

и равны

$$
a=b=-\frac{5}{3}, \quad c=\frac{25 \alpha^{2} \mp \sqrt{600 \alpha^{4}+1296 \lambda^{4}}}{36 \alpha^{2}}, \quad d=\frac{25 \alpha^{2} \pm \sqrt{600 \alpha^{4}+1296 \lambda^{4}}}{36 \alpha^{2}} .
$$

Выражение для функции $X(\xi)$ примет вид

$$
\begin{gathered}
X(\xi)=(1+\alpha \xi)^{2 / 3}\left(C_{1} \sin (\delta \cdot \ln (1+\alpha \xi))+C_{2} \cos (\delta \cdot \ln (1+\alpha \xi))+\right. \\
+C_{3} \operatorname{sh}(\delta \cdot \ln (1+\alpha \xi))+C_{4} \operatorname{ch}(\delta \cdot \ln (1+\alpha \xi))
\end{gathered}
$$

где $\delta=\frac{\sqrt[4]{600 \alpha^{4}+1296 \lambda^{4}}}{6 \alpha}$.

Рассмотрим два вида граничных условий: жесткое закрепление и свободное опирание краёв оболочки.

В случае жесткого закрепления краёв граничные условия имеют вид

$$
u=0, \quad v=0 \quad \text { при } \quad \xi=0, \quad \xi=1
$$

или

$$
X(\xi)=0, \quad \frac{d X(\xi)}{d \xi}=0 \quad \text { при } \quad \xi=0, \quad \xi=1 .
$$

Если края оболочки свободно оперты, то следует удовлетворять условиям вида

$$
u=0, \quad T_{1}=0 \quad \text { при } \quad \xi=0, \quad \xi=1
$$

или

$$
X(\xi)=0, \quad \frac{d^{2} X(\xi)}{d \xi^{2}}=0 \quad \text { при } \quad \xi=0, \quad \xi=1 .
$$

Подчиняя общее решение уравнения граничным условиям и требуя совместности системы однородных уравнений, получаем соотношения для определения собственных значений для жесткого закрепления в форме

$$
\delta \ln (1+\alpha)=4.73
$$

а в случае свободного опирания краев - в форме

$$
\sin (\delta \ln (1+\alpha))=0 .
$$

Для очень длинных оболочек $(R / l \rightarrow 0)$ величина $q_{0}$ нечувствительна к виду закрепления и равна

$$
q_{0}=\frac{E h_{0}^{3}\left(k^{2}-1\right)}{R^{3} 12\left(1-\nu^{2}\right)}
$$

После минимизации по $k^{2}\left(k^{2} \gg 1\right)$ для оболочек средней длины получаем формулу для вычисления критического давления:

$$
q_{0}^{*}=\sqrt[4]{\beta_{0}} E\left(\frac{h_{0}}{R}\right)^{5 / 2} \frac{R}{l} \frac{\sqrt{6}}{9\left(1-\mu^{* 2}\right)^{3 / 4}} \sqrt[4]{\frac{n_{2}^{3}}{m_{1}}}
$$


где величина $\beta_{0}$ в случае жесткого закрепления равна

$$
\beta_{0}=\left(\frac{4.73 \alpha}{\ln (1+\alpha)}\right)^{4}-\frac{75}{162} \alpha^{4}
$$

а в случае свободного опирания краев -

$$
\beta_{0}=\left(\frac{\pi \alpha}{\ln (1+\alpha)}\right)^{4}-\frac{75}{162} \alpha^{4}
$$

$\mu^{*}=\mu / n$.

Формулу (6) можно переписать для жесткого закрепления краев в форме

$$
q_{0}^{*}=4.73 E K(\alpha)\left(h_{0} / R\right)^{5 / 2} \frac{R}{l} \frac{\sqrt{6}}{9\left(1-\mu^{*} 2\right)^{3 / 4}} \sqrt[4]{\frac{n_{2}^{3}}{m_{1}}},
$$

а для свободного опирания торцов соответственно в виде

$$
q_{0}^{*}=\pi E K(\alpha)\left(h_{0} / R\right)^{5 / 2} \frac{R}{l} \frac{\sqrt{6}}{9\left(1-\mu^{*} 2\right)^{3 / 4}} \sqrt[4]{\frac{n_{2}^{3}}{m_{1}}} .
$$

Значения коэффициента $\mathrm{K}(\alpha)$ для некоторых значений $\alpha$ приведены в таблице.

\begin{tabular}{|c|c|c|c|c|c|c|c|c|c|c|c|}
\hline$\alpha$ & -0.5 & -0.4 & -0.3 & -0.2 & -0.1 & 0 & 0.1 & 0.2 & 0.3 & 0.4 & 0.5 \\
\hline $\mathrm{K}(\alpha)$ & 0.750 & 0.798 & 0.847 & 0.888 & 0.950 & 1 & 1.049 & 1.093 & 1.136 & 1.173 & 1.20 \\
\hline
\end{tabular}

На основании полученных результатов можно сделать следующий вывод.

Значения критического давления неоднородной цилиндрической оболочки под действием неравномерного давления, изменяющегося по закону изменения жесткости, пропорциональны критическому давлению оболочки постоянной толщины с коэффициентом пропорциональности $K(\alpha)$, и при увеличении отношения толщин $h_{1} / h_{0}$ на концах оболочки критическое давление уменьшается, а при его уменьшении критическое давление увеличивается.

\title{
Библиографический список
}

1. Власов В. З. Общая теория оболочек. М. : ГИТТЛ, 1949. $784 \mathrm{c}$.

линдрической оболочки переменной толщины // Изв. вузов. Машиностроение. 1975. № 11. С. 27-31.

2. Бурмистров Е. Ф. Симметричная деформация конструктивно-ортотропных оболочек вращения. Саратов : Изд-во Сарат. ун-та, 1962. 108 с.

4. Штейнберг М. В. Расчет круговых цилиндрических оболочек с толщиной переменной в направлении образующей // Прикладная механика. 1965. Т. 1, вып. 7.

3. Мочалин A. А. Устойчивость полубезмоментной ци-

C. $67-72$.

\section{The Stability of the Constructive-orthotropic Heterogeneous Cylindrical Shell under Uneven Radial Load}

\begin{abstract}
A. A. Mochalin
Saratov State Technical University, 77, Polytechnicheskaya str., 410054, Saratov, Russia, a.mochalin@inbox.ru

On the base haft-momentum Vlasov theory the problem of stability of cylindrical homogeneas shell with variation of thicknees atv radial symmetrical ractial pressure variated onalong axe distance. At one reletion between thickness and pressure values the accurate solution was produced for one values in pressure variation law when stability of shell is sailed.

Key words: cylindrical shell, theory of shells, stability of shells, radial loading, critical pressure, shell thickness, the theory of elasticity.
\end{abstract}




\title{
References
}

1. Vlasov V. Z. Obshchaia teoriia obolochek [General 3. Mochalin A. A. Sustainability semi-momentless cylintheory of shells]. Moscow, Gos. izd. teh. teor. lit., 1949, drical shell of variable thickness. Izv. vuzov. Mashino784 p. (in Russian).

2. Burmistrov E. F. Simmetrichnaia deformatsiia konstruktivno-ortotropnykh obolochek vrashcheniia [Symmetric deformation of structurally orthotropic shells of revolution]. Saratov, Saratov. Univ. Press, 1962. 108 p. (in Russian). stroenie, 1975, no. 11, pp. 27-31 (in Russian).

4. Shteinberg M. V. Calculation of circular cylindrical shells with variable thickness in the direction of forming. Prikladnaia mekhanika, 1965, vol. 1, iss. 7, pp. 67-72 (in Russian).

УДК 539.274

\section{РАСЧЕТ ПАРАМЕТРОВ НАГРУЖЕНИЯ ПОЛОГО ШАРА В УСЛОВИЯХ БОЛЬШИХ УПРУГОПОЛЗУЧИХ ДЕФОРМАЦИЙ}

\section{Е. В. Мурашкин}

\begin{abstract}
Кандидат фризико-математических наук, научный сотрудник, Институт проблем механики им. А. Ю. Ишлинского РАН, Москва, murashkin@ipmnet.ru, evmurashkin@gmail.com

Предложена модель больших упругоползучих деформаций. Разделение тензора полных десрормаций альманси определяется уравнениями изменения обратимой и необратимой его составляющих. Рассмотрено ссрерически симметричное десрормирование полого шара в процессе установившейся ползучести. Получена разрешающая система уравнений рассматриваемой краевой задачи. Предложен способ определения нагружающего усилия вызывающего заданное десрормированное состояние. По заданным законам изменения поля перемещений построены фрункции внешнего нагружающего усилия.
\end{abstract}

Ключевые слова: большие десрормации, ползучесть, упругость, релаксация.

\section{ВВЕДЕНИЕ}

Необходимость повышения точности математического описания процессов, происходящих при технологической обработке и эксплуатации металлоизделий, вынуждает учитывать упругие свойства материалов на всех стадиях жизненного цикла изделия. Рассмотрение задач в классических моделях малых деформаций невозможно, когда относительное изменение формы рассматриваемого тела велико. Одной из таких характерных задач, где нельзя обойтись без применения модели больших деформаций, является задача о моделировании процессов в окрестности микропоры в металле, происходящих под действием интенсивного давления. Актуальность данной задачи обусловлена обнаруженным на опыте эффектом существенного повышения эксплуатационных характеристик металла при интенсивном всестороннем сжатии образцов [1] «залечивания» микродефектов сплошности. Попытки смоделировать процесс залечивания микропоры в металле делались неоднократно, в том числе и на основе модели больших упругопластических деформаций [2], обладающей эффектом приспосабливаемости к периодическим нагружениям по циклу «нагрузка - разгрузка» [3].

В настоящей работе решена задача о сферически симметричном сжатии шара с микропорой в центре. Условие несжимаемости среды определяет кинематику среды с точностью до неизвестной функции времени, что позволяет по известному закону деформирования определить процесс нагружения, вызывающий заданное деформированное состояние.

\section{1. ОСНОВНЫЕ МОДЕЛЬНЫЕ ЗАВИСИМОСТИ}

За основу возьмем модель больших упругопластических деформаций [3], основные кинематические соотношения которой в прямоугольной декартовой системе пространственных (эйлеровых) 